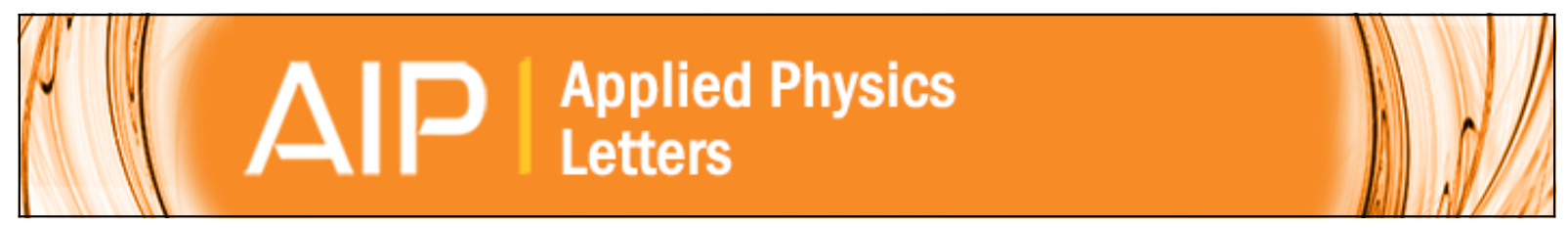

Nature of defects for bismuth layered thin films grown on Pt electrodes

A. Z. Simões, L. S. Cavalcante, E. Longo, J. A. Varela, C. S. Riccardi, and B. Mizaikoff

Citation: Applied Physics Letters 90, 082910 (2007); doi: 10.1063/1.2472527

View online: http://dx.doi.org/10.1063/1.2472527

View Table of Contents: http://scitation.aip.org/content/aip/journal/apl/90/8?ver=pdfcov

Published by the AIP Publishing 


\title{
Nature of defects for bismuth layered thin films grown on Pt electrodes
}

\author{
A. Z. Simões, L. S. Cavalcante, ${ }^{\text {a) }}$ E. Longo, and J. A. Varela \\ Universidade Estadual Paulista, P.O. Box 355, Araraquara, SP 14801-907, Brazil \\ C. S. Riccardi and B. Mizaikoff \\ Georgia Institute of Technology, 901 Atlantic Drive, Atlanta, Georgia 30332-0400
}

(Received 8 November 2006; accepted 10 January 2007; published online 23 February 2007)

\begin{abstract}
The authors investigated the influence of defects on the piezoelectric and dielectric properties of $\mathrm{Bi}_{4} \mathrm{Ti}_{3} \mathrm{O}_{12}$ (BIT), $\quad \mathrm{SrBi}_{4} \mathrm{Ti}_{4} \mathrm{O}_{15} \quad(\mathrm{SBTi})$ and $\mathrm{CaBi}_{4} \mathrm{Ti}_{4} \mathrm{O}_{15} \quad$ (CBTi144) thin films by $\mathrm{X}-$ ray photoemission spectroscopy measurements. In the SBTi film, Sr which is a nonpolarizable ion restricting the movement of $\mathrm{Ti}^{4+}$ ions and thus leads to a low piezoresponse. Meanwhile, the oxygen environment is quite different in the BIT and CBTi144 films exhibiting excellent piezoelectric properties. The piezoelectric coefficient and the dielectric behavior were larger for $a-b$ axis oriented than for $c$ axis-oriented films due to the defects created during the films crystallization. (C) 2007 American Institute of Physics. [DOI: 10.1063/1.2472527]
\end{abstract}

Bismuth-based layer-structured ferroelectrics are attractive materials because of their high potential for application to ferroelectric random access memories, lead-free piezoelectric resonators, and dynamic random access memories. ${ }^{1,2}$ $\mathrm{Bi}_{4} \mathrm{Ti}_{3} \mathrm{O}_{12}$ (BIT) is a promising candidate as an alternative material because of its large spontaneous polarization. Also, higher order structures such as $\mathrm{SrBi}_{4} \mathrm{Ti}_{4} \mathrm{O}_{15}$ (SBTi) and $\mathrm{CaBi}_{4} \mathrm{Ti}_{4} \mathrm{O}_{15}$ (CBTi144) may have larger remanent polarization due to the increased number of perovskite units generating ferroelectric properties. SBTi, which has a crystalline structure similar to BIT, is another typical layer-structured ferroelectric $(m=4))^{3}$ Its high Curie temperature $\left(T_{C}\right.$ $=520^{\circ} \mathrm{C}$ ) makes it useful over a wide temperature range. ${ }^{4,5}$ However, the $2 P_{r}$ of SBTi thin films is lower $\left(6.2 \mu \mathrm{C} / \mathrm{cm}^{2}\right.$, compared to $\left.13 \mu \mathrm{C} / \mathrm{cm}^{2}\right),{ }^{6-8}$ and the fatigue endurance property deteriorates with the increase of switching pulse width. CBTi144 is a member of the Aurivillius family, layerstructured ferroelectrics. Also, CBTi144 is characterized at its high Currie point $\left(T_{C}\right)$ of about $790{ }^{\circ} \mathrm{C}$. ${ }^{9,10}$ Therefore, it is expected for special applications at relatively high temperature. X-ray photoemission spectroscopy (XPS) is a nondestructive method, which is sensitive to local electronic structure of constituent atoms and is among the most suitable technique for investigating the effects of surface layers on the polarization properties and piezoelectric behavior of polycrystalline ceramics. $^{11}$

In this letter, XPS studies were undertaken on various bismuth-layered compounds in order to probe their surface characteristics and to explore the nature of defects in the crystal lattice. $\mathrm{CaCO}_{3}$ (Aldrich), $\left(\mathrm{Ti}\left[\mathrm{OCH}\left(\mathrm{CH}_{3}\right)_{2}\right]_{4}\right)$ (Hulls$\mathrm{AG}$ ), $\mathrm{Bi}_{2} \mathrm{O}_{3}$ (Aldrich), and $\mathrm{SrCO}_{3}$ (Aldrich) were used as raw materials. The preparation of precursor solutions is described elsewhere. ${ }^{12}$ The BIT, SBTi, and CBTi144 thin films were spin coated on $\mathrm{Pt}(100) / \mathrm{Ti} / \mathrm{SiO}_{2} / \mathrm{Si}$ substrates by a commercial spinner operating at $5000 \mathrm{rpm}$ for $30 \mathrm{~s}$ (spin coater $\mathrm{KW}-4 \mathrm{~B}$, Chemat Technology). The thin films were annealed at $700{ }^{\circ} \mathrm{C}$ for $2 \mathrm{~h}$ in a conventional furnace. Through this process, we have obtained thickness values of about $300 \mathrm{~nm}$ for all systems. Phase analysis was performed at room temperature by X-ray diffraction (XRD) in the $\theta-2 \theta$

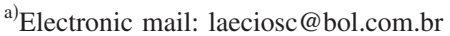

mode scan using $\mathrm{Cu} K \alpha$ radiation. Furthemore, topography and thickness were examined using atomic force microscopy (AFM) (Digital Instruments, Nanoscope IV) and scanning electron microscopy (Topcom SM-300), respectively. The top $\mathrm{Pt}$ electrodes were prepared by photolithography with 8 $\times 10^{-4} \mathrm{~mm}^{2}$ dot area. The capacitance-voltage characteristic was measured in the metal-ferroelectric-metal configuration using a small ac signal of $10 \mathrm{mV}$ at $100 \mathrm{kHz}$. The ac signal was applied across the sample, while the dc was swept from positive to negative bias. The piezoelectric measurements were done using a setup based on an atomic force microscope in a multimode scanning probe microscope with Nanoscope IV controller.

Figure 1 shows the XRD results of all investigated systems. The SBTi and BIT films present $c$ axis orientation. All the films present an orthorhombic structure at room temperature, with space and number of the perovskite unit cells between $\mathrm{Bi}_{2} \mathrm{O}_{2}$ layers. Meanwhile, CBTi144 films showed a high intensity of the (200)/ (020) diffraction line compared to the other lines, although the (200) and (020) diffraction lines could not be distinguished from each other. The characteristic orientation is considered to be due to good matching of atomic arrangements in CBTi144 (100)/(010) and underlying Pt planes. The lattice constants $a$ (or $b$ ) and $c$ of the CBTi144 film are close to 0.5417 and $4.086 \mathrm{~nm}$, the lattice

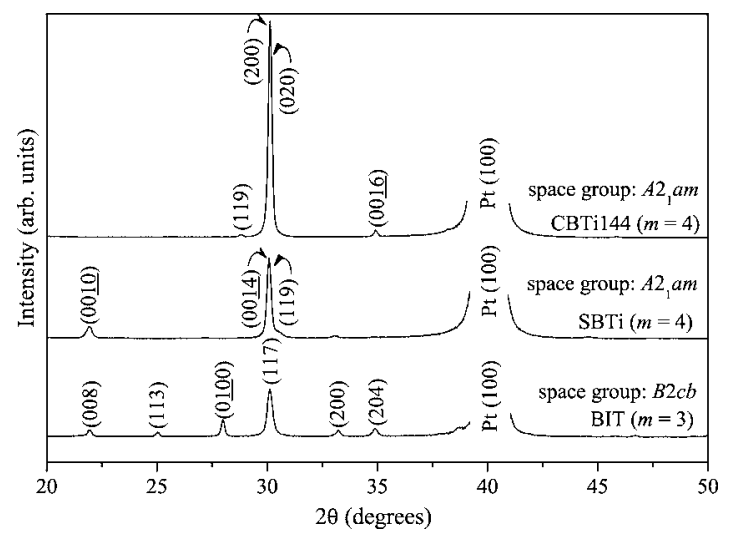

FIG. 1. X-ray diffraction for BIT, CBTi144, and SBTi thin films deposited on $\mathrm{Pt}(100) / \mathrm{Ti} / \mathrm{SiO}_{2} / \mathrm{Si}$ substrate at $700{ }^{\circ} \mathrm{C}$ for $2 \mathrm{~h}$. 


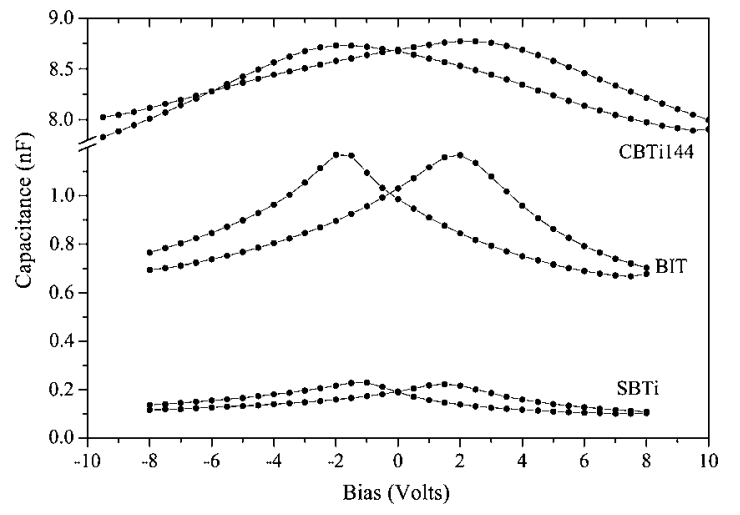

FIG. 2. $C$ - $V$ curves for BIT, CBTi144, and SBTi thin films deposited on $\mathrm{Pt}(100) / \mathrm{Ti} / \mathrm{SiO}_{2} / \mathrm{Si}$ substrate at $700{ }^{\circ} \mathrm{C}$ for $2 \mathrm{~h}$.

mismatches between the ferroelectric phase and Pt were given remarkably small as $1.1 \%$ and $4.9 \%$, respectively. The characteristic $(100) /(010)$ orientation of the CBTi144 film is based on the good lattice matching of CBTi144 (001) with Pt planes.

The $C$ - $V$ curves indicate two peaks (see Fig. 2). Also, the curve displays symmetry in the maximum capacitance values that can be observed in the vicinity of the spontaneous polarization switching. From $C-V$ the measured value of relative dielectric constant at a frequency of $1 \mathrm{MHz}$ is 265 for BIT, 35 for SBTi, and 1535 for CBTi144 films. As we expected, the lower value of dielectric constant is related to the contribution of $c$ axis orientation and the nature of defects.

The $d_{33}(V)$ hysteresis loops are shown in Fig. 3. The $d_{33}$ value of BIT is close to $40 \mathrm{pm} / \mathrm{V}$ and approaches the reported value for a BIT single crystal. ${ }^{11}$ An enhancement of piezoelectric coefficient was observed for the CBTi144 film approaching a maximum value of $65 \mathrm{pm} / \mathrm{V}$, which is close to the reported value for the single crystal. ${ }^{13}$ The enhancement could be caused by the $a / b$ axis orientation of the ferroelectric films due to the preferred orientation of the $\mathrm{Pt}$ substrate. As can be seen, the piezoloop shows an offset in the vertical direction which is probably caused by the clamping effect and the existence of an ultrathin air gap between the tip and the sample, which might lower the actual voltage drop across the film. ${ }^{14,15}$ Meanwhile, SBTi thin films present a typical dielectric behavior and therefore translate to a weak piezoelectric response. The pulsed force mode (PFM) images

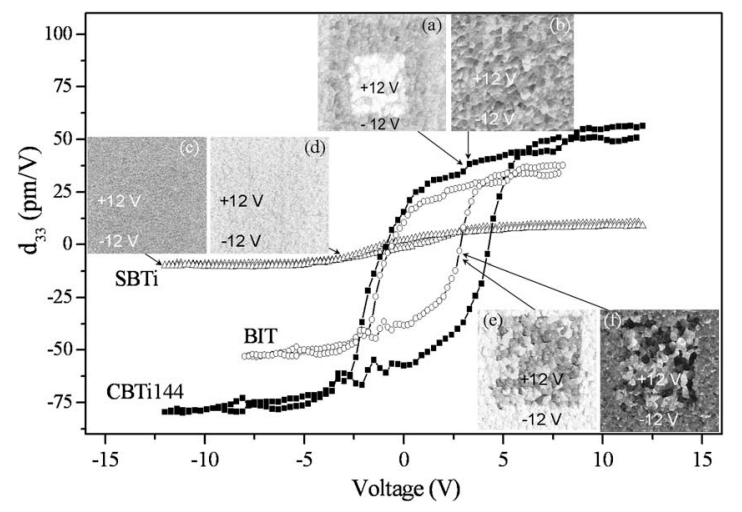

FIG. 3. Piezoresponse loops and domain images obtained for bismuth layered thin films deposited on $\mathrm{Pt}(100) / \mathrm{Ti} / \mathrm{SiO}_{2} / \mathrm{Si}$ substrate at $700{ }^{\circ} \mathrm{C}$ for $2 \mathrm{~h}$. The inset shows (a) CBTi144 out of plane view, (b) in-plane view, (c) SBTi out of plane view, (d) in-plane view, (e) BIT out of plane view and (f) in-plane view. of BIT, SBTi, and CBTi144 thin films are also illustrated. Here, the negative pole of the $12 \mathrm{~V}$ power supply was applied to the substrate bottom electrode in order to check the piezoelectric activity. Two regions with different contrasts can be distinguished in the BIT and CBTi144 thin films; the light contrast corresponds to the as-grown film, the dark one arose upon application of the dc voltage, which results in a polarization vector along the electric field, pointing perpendicular into the film plane. In the out-of-plane image [see the inset in Figs. 3(a, c, and e)], the color contrast indicates domains oriented in direction to the bottom electrode and the AFM tip. The dark region indicates that the polarization vector is oriented in the tip direction. Generally, inside the darker quadratic regions that exhibit a small piezoelectric response, are always unpolarized crystallites (light regions). By changing the polarity of the applied voltage it can be easily demonstrated that the light regions present a polarization vector perpendicular to the film surface, whereas in the dark regions the polarization vector lies in the film plane. The PFM images of SBTi film poled with a negative $-12 \mathrm{~V}$ power supply show no evidence of piezoelectric behavior due the low piezoresponse and the nature of defects created by the strontium ion at the $A$ site of SBTi. On the other hand, a high contrast images were evident for BIT and CBTi144 films, indicating that both preferred orientation and small quantity of oxygen vacancies allow the reverse of piezoelectric domains and thus to piezoelectric activity of those films. In the in-plane PFM images [see the inset in Figs. 3(b, d, and f)] the contrast changes were associated with changes of the in-plane polarization components. In this case, the white contrast indicates polarization, e.g., in the positive direction of the $y$ axis while dark contrast are given by in-plane polarization components pointing to the negative part of the $y$ axis.

The nature of defects in BIT, SBTi, and CBTi144 thin films was investigated by XPS. Photoemissions of $\mathrm{Bi} 4 f, \mathrm{Sr}$ $3 d$, Ca $2 p$, and Ti $2 p$ core levels were performed, and the results are shown in Fig 4. The photoemission spectra of $\mathrm{Bi}$ $4 f$ core level indicate that the $7 / 2$ and $5 / 2$ spin-orbit double components are located approximately at 160.4 and $165.7 \mathrm{eV}$, respectively [Fig. 4(a)]. The spin-orbit splitting of $\mathrm{Bi} 4 f$ core levels is around $5.3 \mathrm{eV}$. The binding energy of $\mathrm{Bi}$ $4 f$ reduces with $\mathrm{Sr}$ addition by about $0.15 \mathrm{eV}$, in contrast to those of CBTi144 and BIT thin films, suggesting the increasing of the chemical valence of $\mathrm{Bi}$ and $\mathrm{Ti}$. That is, to say, the chemical valences of $\mathrm{Bi}$ and $\mathrm{Ti}$ approache +3 and +4 valence states, which implies that the strontium substitution in the $A$ site increases the concentration of oxygen vacancies in the vicinity of $\mathrm{Bi}$ and $\mathrm{Ti}$ ions. When $\mathrm{Sr}$ is added in the system the $\mathrm{TiO}_{6}$ linked to $\mathrm{Bi}_{2} \mathrm{O}_{2}$ shrinks, restricting the movement of $\mathrm{Ti}^{4+}$ ions and thus reducing the space charge compensation. This result is in agreement with the low piezoresponse of SBTi thin films and can be related to oxygen vacancy acting as space charge which causes strong domain pinning and inhibits the poling of the film. The binding energy is not strong influenced by calcium addition, indicating a reduction in the oxygen vacancy concentration in the system. The decrease of oxygen vacancy concentration, competing with the structural changes, brings about the variation of piezoelectric coefficient between BIT, CBTi144, and SBTi films. Comparing with calcium, strontium is a sort of more stable and nonpolarizable ion, and thus causes the decrease of the piezoelectric coefficient. These results indicate that the 

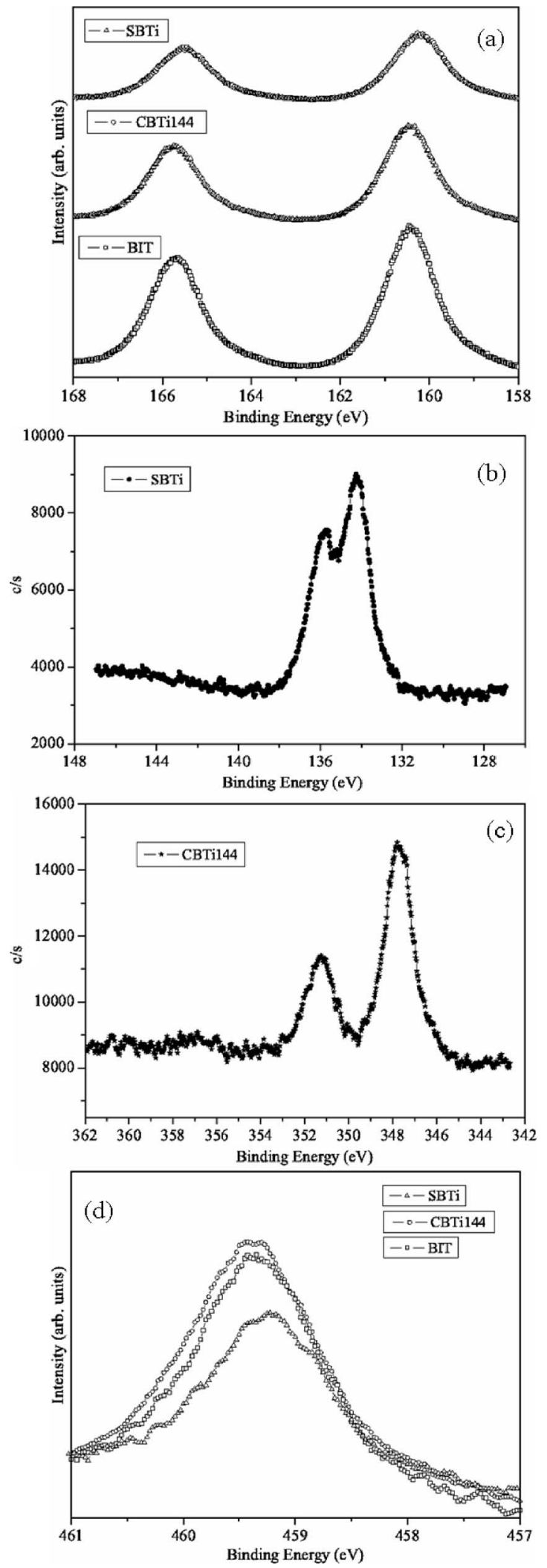

FIG. 4. XPS spectra of (a) Bi $4 f$, (b) $\mathrm{Sr} 3 d$, (c) Ca $2 p$, and (d) Ti $2 p$ peaks for BIT, CBTi144, and SBTi thin films deposited on $\mathrm{Pt}(100) / \mathrm{Ti} / \mathrm{SiO}_{2} / \mathrm{Si}$ substrate at $700{ }^{\circ} \mathrm{C}$ for $2 \mathrm{~h}$.

environment of the oxygen ions near the $\mathrm{Ca}$ and $\mathrm{Bi}$ ions is quite different from that near the Sr ions. Thus, for SBTi thin films, oxygen vacancies seem to be preferably present near the $\mathrm{Bi}$ ions at the $\mathrm{Bi}_{2} \mathrm{O}_{2}$ layers. The $5 / 2$ and $3 / 2$ spin-orbit doublet components of the $\mathrm{Sr} 3 d$ core level photoemission are shown [Fig. 4(b)]. The peaks can be analyzed by two types of spin-orbit doublet: one is the spin-orbit doublet of the $\mathrm{Sr} 3 d$ core level and the other is that in another $\mathrm{Sr}$ oxide different from SBTi. However, the peak positions do not coincide with those of metallic $\mathrm{Sr} 3 d(5 / 2=134.3 \mathrm{eV}$ and $3 / 2=136 \mathrm{eV}) .{ }^{16}$ The photoemission spectra of $\mathrm{Ca} 2 p$ core levels indicates that the $3 / 2$ and 5/2 spin-orbit double components are located approximately at 347.8 and $351.3 \mathrm{eV}$, respectively [Fig. 4(c)]. Identical to $\mathrm{Sr}$ peaks, two types of spin-orbit doublet were evident: one is the spin-orbit doublet of the $\mathrm{Ca} 2 p$ core level and the other is that in another $\mathrm{Ca}$ oxide different from CBTi144.

The photoemission spectra of Ti $2 p$ core levels are also shown [Fig. 4(d)]. The Ti 2p3/2 photoelectron peak appears in the XPS spectrum as a distinctively resolved feature at a binding energy position of approximately $459.3 \mathrm{eV}$. The Ti $2 p$ peaks for the BIT and CBTi144 films are broader than that for the SBTi film, suggesting that some oxygens at the perovskite layers are taken out. These experimental results imply that oxygen vacancies could be induced in the neighborhood of the $\mathrm{Bi}$ and the $\mathrm{Ti}$.

In summary, we investigated the nature of defects on piezoelectric and dielectric properties of several thin films of Aurivillius family obtained by the polymeric precursor method. XPS data reveal that $\mathrm{Sr}$ which is a nonpolarizable ion restricting the movement of $\mathrm{Ti}^{4+}$ ions and thus reducing the space charge compensation leading to a low piezoresponse. Meanwhile, BIT and CBTi144 films exhibited excellent piezoelectric properties, indicating that the environment of the oxygen ions is quite different from that of SBTi films. Both piezoelectric coefficient and dielectric constant were larger for $a-b$ axis oriented than for $c$ axis-oriented films due to the defects created during the films crystallization.

Dr. Hess of the Georgia Institute of Technology is acknowledged for XPS measurements.

${ }^{1}$ D. Wu, A. Li, T. Zhu, Z. Liu, and N. Ming, J. Appl. Phys. 88, 5941 (2000).

${ }^{2}$ Y. Adachi, D. Su, P. Muralt, and N. Setter, Appl. Phys. Lett. 86, 172904 (2005).

${ }^{3}$ M. Hirose, T. Suzuki, H. Oka, K. Itakura, Y. Miyauchi, and T. Tsukada, Jpn. J. Appl. Phys., Part 1 38, 5561 (1999).

${ }^{4}$ H. Lrie and M. Miyayama, Appl. Phys. Lett. 79, 251 (2001).

${ }^{5}$ Y. Noguchi, M. Miyayama, and T. Kudo, Appl. Phys. Lett. 77, 3639 (2000).

${ }^{6}$ S.-T. Zhang, B. Yang, Y.-F. Chen, Z.-G. Liu, X.-B. Yin, Y. Wang, M. Wang, and N.-B. Ming, J. Appl. Phys. 91, 3160 (2002).

${ }^{7}$ S.-T. Zhang, B. Sun, B. Yang, Y. F. Chen, Z. G. Liu, and N. B. Ming, Mater. Lett. 47, 334 (2001).

${ }^{8}$ D. S. Sohn, W. X. Xianyu, W. I. Lee, and I. Chung, Appl. Phys. Lett. 79, 3672 (2001).

${ }^{9}$ E. C. Subbarao, J. Am. Ceram. Soc. 45, 166 (1962).

${ }^{10}$ L. Korzanova, Ferroelectrics 134, 175 (1992).

${ }^{11}$ B. H. Park, S. J. Hyun, S. D. Bu, T. W. Noh, J. Lee, and H. D. Kim, Appl. Phys. Lett. 74, 1907 (1999).

${ }^{12}$ A. Z. Simoes, C. Quinelato, A. Ries, E. Longo, and J. A. Varela, Mater. Chem. Phys. 98, 481 (2006).

${ }^{13}$ A. Saneto and L. E. Cross, J. Mater. Sci. 17, 1409 (1982).

${ }^{14}$ B. S. Kang, B. H. Park, S. D. Bu, S. H. Kang, and T. W. Noh, Appl. Phys. Lett. 75, 2644 (1999).

${ }^{15}$ A. Gruverman, O. Auciello, and H. Tokumoto, Annu. Rev. Mater. Sci. 28, 101 (1998)

${ }^{16}$ J. F. Moulder, W. F. Stickle, P. E. Sobol, and K. D. Bomben, Handbook of $X$-Ray Photoelectron Spectroscopy (Physical Electronics, Eden Prairie, $\mathrm{MN}, 1995)$, p. 105 